\title{
An Analysis of Common Mistakes Seen in Scientific Writing in English by Chinese Biomedical Students
}

\author{
Xiang-Ning ZHANG ${ }^{1,{ }^{*}}$, Bi-Ying ZHENG ${ }^{2}$, Gan HOU ${ }^{3}$ and Ruo-Lin ZHANG ${ }^{4}$ \\ ${ }^{1}$ Department of Pathophysiology, School of Basic Medical Science, \\ Guangdong Medical University, \\ Songshan Lake Scientific Park, Dongguan, Guangdong 523808, China \\ ${ }^{2}$ Departments of Microbiology, School of Laboratory Medicine, Guangdong Medical University, \\ Songshan Lake Scientific Park, Dongguan, Guangdong 523808, China \\ ${ }^{3}$ Departments of Biochemistry, School of Laboratory Medicine, Guangdong Medical University, \\ Songshan Lake Scientific Park, Dongguan, Guangdong 523808, China
}

${ }^{4}$ Class 2, Enrolment 2015, Department of English, Sontan College, Guangzhou University, Zengcheng, Guangzhou, Guangdong 510000, China

*zhangxn_2006@126.com

Keywords: Scientific writing, Medical terminology, Binomenial nomenclature, Model organism.

\begin{abstract}
To improve the writing ability on scientific papers for biomedical students, and meet the demand of academic communication in their future career, we launched a course for the final year students of laboratory medicine, who had returned from clinical internship, and were drafting their baccalaureate degree thesis. The medical periodicals relevant to the study field of the students were introduced, and the English abstracts of the students' thesis were examined. During the teaching, mistakes frequently made have been identified. These included the information asymmetry in bilingual expression, the inappropriate use of non-medical terms in the scientific papers, and the incorrect font of binomen of organisms. The problems should be solved with the English teaching at universities together with the introduction of bilingual instruction. Meanwhile the amendment of the nomenclature in species should be paid attention to by scientific workers, and a life time learning is proposed to our students.
\end{abstract}

\section{Introduction}

With the increase of the financial input in scientific research, the overall research quality in China has been remarkably improved. For the purpose of international communications, English becomes frequently used in the regular scientific writting. This also includes composing English abstract for scientific papers in Chinese. To meet such a demand, the school of laboratory medicine of our university has designed a course of scientific writing in English, for the final year students returning from their clinical internship. The course has been administered for three years. During the course, the English abstract of the students' baccalaureate thesis was examined, and common problems for the writing of scientific papers in English have been identified. They were summarized in the present paper, in order to attract the attentions of the faculty of different subjects, and to specifically solve the issues during teaching of different courses. The problems are listed below.

\section{Frequent Mistakes Seen in the Scientific Writing of the Biomedical Students}

\section{Asymmetry of Information within Titles in Chinese and English}

The problem may be due to the less careful translation. The thesis of a student dealt with the detection of hepatitis virus proteins in the serum samples of a group of patients. The amount of samples, 188 was clearly stated in the Chinese title, in line with the requirement of title of medical writing. The title in English, however, became " Analysis of hepatitis B virus large surface protein”; 
the key information was disappeared. The amount of the samples would clearly suggest the degree of reliability of the investigation. In the writing of the subjects to enter a clinical study, involving " within a specific time interval”, “ a given number of patients”, "diagnosed with a clinical entity”, " were admitted to a hospital", the illogical arrangement of these elements are frequently witnessed in the degree thesis. The structural analysis of long sentence in English should be paid much attention in teaching of basic English, in avoidance of the some errors. Another student discussed about the overlapping infection of superinfection of Hepatitis B and E, with an acceptable title in Chinese. The title in English was "Hepatitis E virus infection and overlapping Hepatitis types B and E viruses infection". In fact, he had chosen a term "superinfection" as a keyword, with correct writing in English, but the word did not appear in the English title. It is suggested that the word "overlapping...infection" be replaced by "superinfection", which is defined as new infection occurring in individuals with preexisting infection, for example, a patient with infection of respiratory virus to be infected by a bacterium, or a chronic hepatitis B patient is infected by hepatits $\mathrm{E}$ [1]. Apparently the term appropriately reflects the clinical process that the patients sequentially infected with two types of hepatitits virus. Superinfection is clinically frequent; for example, the infection of different strains of human immune deficient virus (HIV) on the same host [2]. A lymphotropic human herpesvirus, Epstein-Barr virus (EBV), normally infect human monoclonally; the infection of multiple strains could be diagnosed by detection of the viral antigens or some repetitive DNA sequences in the viral genome [3, 4].

\section{Inappropriate Use of Non-biomedical Term in Writing}

A student analyzed the allergens in allergic patients in Shenzhen region, southern China. One of the food allergen was "egg white". In scientific writing, the word to be used should be "albumen", which refers to the viscous and clear liquid enclosed by the outer membrane inside the shell of the egg. The word has one letter difference of "albumin", the major protein component of "albumen". In this context, both words could be used when referring to the allergen.

In order to improve writing quality of scientific English, it becomes necessary that students should adapt to the thinking way in English through regular reading, to avoid taking something for granted. In English, there is "egg white ", but there is no "egg yellow", like in Chinese. The word is "yolk", like in "yolk sac" in animal embryo. In English, the egg of birds, or peptiles, whether the shell is flexible or hard, together with the oocyte of mammalian, are termed with the same word "egg". This is quite different from Chinese. In scientific papers, however, some less important terms used in the context, could be expressed in non-scientific way. An example, if the body component is discussed the word "parasite" could be replaced by "worm”. If a specific molecule or gene of the insect Drosophila, scientifically called Drosophila melangaster is dealt with, but not the behavior of the entire insect, it could be called "fruitfly.

To develop the language skills through regular reading, is regarded as an effective pathway to improve the students' writing ability. For biomedical students, the contents of their reading should include relevant scientific periodicals, as well as the manual of the instruments and reagents accessible in the labs during their internship of clinical practice. We have found that some students were unable to write correctly the name of the reagent suppliers, like bioMerieux. This may also reflect that they lack the essential knowledge of the experimental materials they are using. In our course for writing English for the laboratory medicine students, we have introduced to them the biomedical periodicals that they could use in their professional work, with emphasis of the publications launched by Society of General Microbiology and American Society for Microbiology, especially the periodicals with the scope in isolation and identification of micobes, their classification and nomenclature, antibiotic resistance, together with infection and immunity. A recent survey was conducted in 266 students after finish of the course, enquiring whether the lecturing could be helpful for them. The result was to be score 1 to 5 , with 5 point being the highest. The data is referred to Fig.1. 


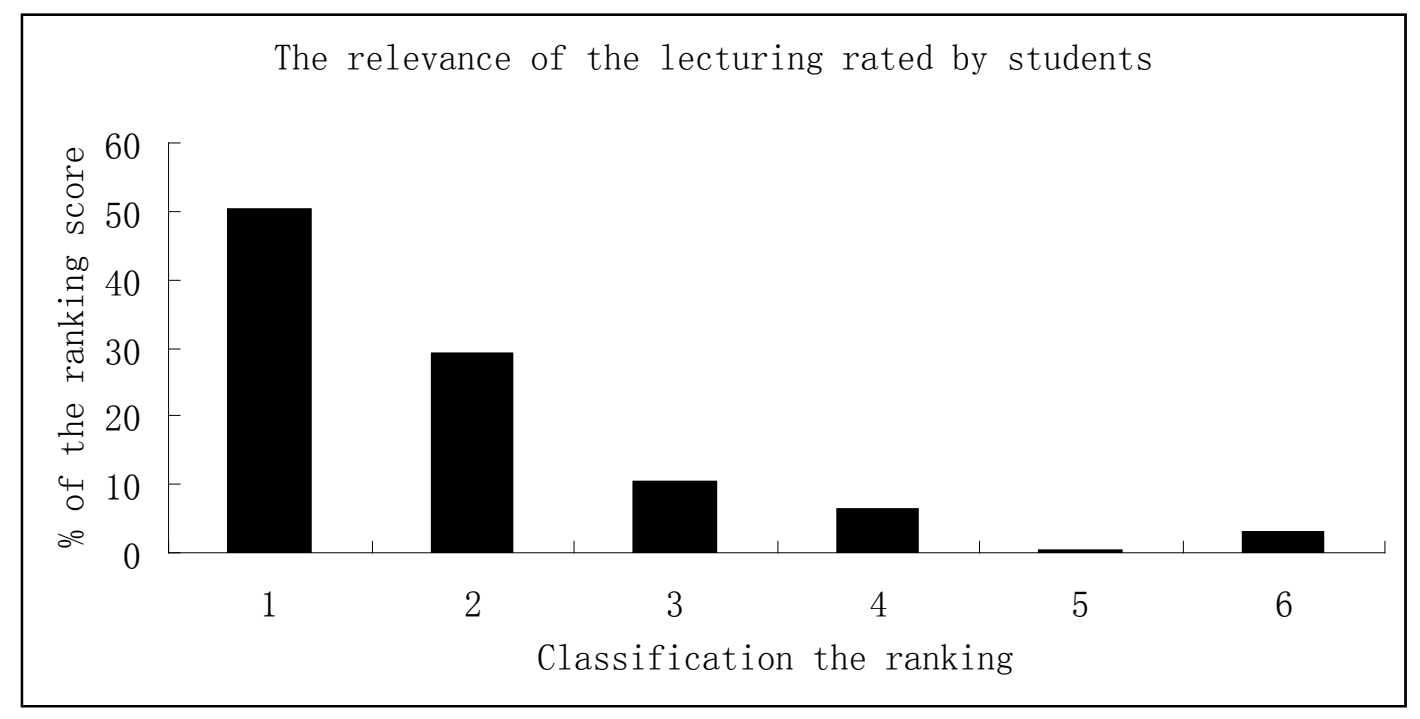

Figure. 1 The survey on helpfulness of lecturing on scientific periodicals to the laboratory medical students. 1. Exceptionally helpful, score 5; 2. very helpful; score 4; 3. helpful score 3; 4. hardly helpful; score 2; 5.not helpful score 1; 6 . No answer.

The data above suggested that over $90 \%$ surveyed students agreed that the lecturing was helpful to them to different degree.

\section{The Writing of Scientific Names with Binomen}

More than three centuries ago, Carl Linnaeus, a Swedish botanist introduced binomen system to name biological species. According to his system, all living things in the world are defined with name of genus in Latin and name of species in genitive case of Latin or Latinized words [5]. For example, the scientific name of "human" is "homo sapiens", which is the species Homo sapiens under the genus Homo. The genus also includes other species like Neanderthal man (Homo neantherdalesis King 1864) [6]. The scientific names are often ended by a capital latter or surname, to suggest the name of the person who name this species. Many scientific name end with a letter L., standing for "Linnaeus". From each scientific name in binomen, such information on who defined the species can be obtained. A plant, Hong Kong orchid was termed scientifically Bauhinia blakeana Dunn. It was named in Hong Kong in the beginning of 20 century, and the species name"blakeana" was derived from the name of a British botanist Sir Henry Blake, who had served as the governor of the colony, and the species was named by a botanist Stephen Troyte Dunn, in Pokfulam, Hong Kong 1908[7]. After the proposal of binomen system, the naming of organisms have been formalized, and, with the amendment of numerous names by various international organizations, currently used names become more and more scientific, greatly facilitating the academic exchanges between countries in the world. The errors seen in the writing of binomen names in scientific papers of biomedical students include:

\section{A Common Mistake is the Incorrect Use of Normal Font when Writing Scientific Names}

Some working books in linguistics define the use of italic type as, to be emphasized [8]. The context includes, name of books. In printing matters, italic type is equivalent with underlining in handwritten. In addition, the abbreviated Latin words, like e.g.( exampli gratia), etc (et cetera); i.e. (id est) are arranged in italics. Italics are also applied in non-English words that appear in text, for example, binomen of living organisms written in Latin [9]. Such names like Acinetobacter baumannii should not appear as normal font in papers. It is noteworthy that for years some students knew and followed the rules. In instruction for authors of medical periodicals, detailed rules of the use of italics are defined. An example, a journal, Emerging Infectious Diseases published by Center for Disease Control and Prevention, set a guideline for writing of names of microbes [10]. The classification units above "genus", the words should be of normal fonts; The scientific name at level 
of "genus" and "species" can be typed in italics; and these two parts are the two parts of a binomen. The unit under species, should also type in normal font. A pathogenic microbe is written as Salmonella enterica typhimurium (serovar). A well known bacterial pathogen Salmonella typhimurium, has been amended from a species to a serotype.

\section{No Separation of the Two Parts of a Binomen, or Only One Part is Presented}

A student presented his thesis on the evaluation of quality of several commercial mycoplasma detection kits. One of the keywords was "Mycoplasmahominis". He knew that the the scientific name of a microbe should be type in italic, but did not separate the two parts of binomen, The correct form should be” Mycoplasma hominis”. Another example, a medical institution in Guangdong surveyed the rodent transmission vectors of hemorrhagic fever with renal syndrome in counties surrounding the city of Guangzhou. Two animals were identified; they were Rattus norvegicus and Rattus losea". When the data was presented to an academic conference, the names of the two rodents became" Rattus norvegicus and losea" As scientific names of animals, the writing should follow the rules of binomen; the name of the genus should not be omitted, even if the two share genus name..It was mentioned in instructions for authors of Emerging Infectious Diseases that, when multiple members of a genus are cited, after the first one being full, the genus of the others can be abbreviated with a capital letter. The two above-mentioned rodents could be written as"Rattus norvegicus and $R$. losea". In fact, the genus of well known species can be abbreviated; like letter " $P$ " in $P$. aeruginosaz represents"pseudomonas “ ; Escherichia coli, which is frequently used in molecular biology as vector of gene expression is often shortened as E. coli.

\section{The Use of Latin Names in Biomedical Science}

Medical terms are written in Latin, in the context of pathogens, of microbial and parasitic, some vectors which transmit diseases, and model organisms for the study of disease and genes. Traditionally, some anatomic terms are also written in Latin, for example locus ceruleus. In most occasions, attention must be paid in choice of text font.

\section{Microbial and Parasitic Pathogens of Human Diseases are Written in Latin Scientific Names}

During our teaching of the medical English teaching, we introduced to the students the major medical periodicals, relating to their future professional career. An example was International Journal of Systematic and Evolutionary Microbiology.As previously mentioned, the names of many microbes are constantly amended, and this should hold our attentions. At the end of 1980s; Chinese cases of a tropical disease, melioidosis were first identified in southern China [11]. The disease was first documented in the beginning of 20th century by a pathologist of British Army, Dr Alfred Whitmore in a place currently as a part of Myanmar[12]. The reported case then was clinically manifested as glanders, and the isolated bacterium was classified under the genus Bacillus, termed "Bacillus pseudomallei". In 1957, it was renamed "Pseudomonas pseudomallei" because of its similarity with Pseudomonas aeruginosa in phenotypes and antibiotic sensitivity. A new genus, Burdkhadia, was proposed in 1993, and P. pseudomallei and P. cepaciae were moved to the new genus [13]. The pathogen of melioidosis has been termed Burdkhadia pseudomallei ever since. The example prompts that much attention should be paid to the latest advance in each field of the career, and life time learning is hence proposed.

\section{The Transmission or Reservoir Vector of Some Diseases}

The examples are the insects Aedes aegypti and A. albopictus, which transmit the arboviral pathogens of dengue fever, yellow fever and Chikungunya viruses to human individuals. And Rattus novergicus and Rattus losea mentioned above. For the binomen scientific names, some species names are in fact identical to the common names of the animal or plant in Latin. Like binomen of domestic cattle, Bos taurus, "taurus" is cattle or cow in Latin, and also an astrological zodiac. But the genus name is a scientific term. There are other names, whose part of genus is a common name. It is likely 
that the name of genus was derived from a type species under it. An example, the genus Rattus, means "rat" in Latin, corresponding with "rat" in English and French, and "Ratte“ in German. A well known species under this genus is $R$. norvegicus, brown rat or Norwegian rat, and the SD rat commonly used in biomedical experiments is its subspecies. The type species under the Rattus is less familiar to Chinese people; it i s a black rat, Rattus rattus originating in Southeast Asia and was spread to the British Isle during the era of ancient Rome [14 ].

\section{Model Organisms for the Study of Diseases and Genes}

The hosts of the sequences of genes and proteins deposited at National Center of Biotechnology Information (NCBI), including human are cited with binomens in Latin. In the system, zebrafish is written as Danio rerio. For the reproducibility of the experimental result, and the purpose of academic exchange in the agricultural study of plants, Arabidopsis thaliana is used as a standard material,but one should not use a similar plant purchased from the supermarket. White leghorn is normally used as the source of chicken embryo when investigation on regulation of angiogenesis is conducted [15]. During the preparation of biomedical research papers, the Journal of General Virology and other periodicals of Microbiology Society require that in the part of methods, detail should be sufficient to allow the same experiment could be repeated[16].

\section{Summary}

With the demand of the current situation of training students of biomedical science, it is suggested that measures should be taken to improve their level of scientific writing in English in the teaching of different courses. The bilingual instruction of biomedical courses at our university has been well meet such requirements; but further efforts are needed to attain a well accepted level.

\section{Acknowledgement}

The work described within has been supported by the Excellence of Quality project of Guangdong Medical University, Bilingual Educational Instruction in Pathophysiology SY 11001. We appreciate the enthusiasm of our students in participation of the survey and their activities in the course.

\section{References}

[1] Miller-Keane Encyclopedia and Dictionary of Medicine, Nursing, and Allied Health. 7th Edition. Saunders, an imprint of Elsevier, Inc.

[2] T.M. Allen, M. Altfeld M. HIV-1 superinfection. J Allergy Clin Immunol. 112(2003):829-35

[3] D.M. Walling, A. L.Brown, W. Etiene, W. A.Keitel, P. D. Ling. Multiple Epstein Bar virus infection in healthy individuals. J Virol. 77(2003): 6546-50

[4] K. Falk, J.W. Gratama, M. Rowe, J.Z. Zou, F. Khanim, L.S. Young, M.A. Oosterveer, I. Ernberg. The role of repetitive DNA sequences in the size variation of Epstein-Barr virus (EBV) nuclear antigens, and the identification of different EBV isolates using RFLP and PCR analysis. J Gen Virol. 76 ( 1995):779-90.

[5] J. David, G.M. Garrity, W. Greuter, D.L. Hawksworth, R. Jahn, P.M. Kirk, J. McNeill, E. Michel, S. Knapp, D. J. Patterson, B.J. Tindall, J.A. Todd, J. van Tol, N.J. Turland. Biological nomenclature terms for facilitating communication in the naming of organisms. Zookeys. 192(2012):67-72.

[6] J.J. Hublin. The origin of Neandertals. Proc Natl Acad Sci USA. 106 (2009): 16022-7

[7] S.T. Dunn. New Chinese Plants. J Botany, British \& foreign. 46 (1908): 324-326. 
[8] L. Truss, Eats, shoots and Leaves. The zero tolerance approach to punctuation. Gotham Books, New York, 2004 (ISBN 1-59240-087-6).

[9] Italic type on en.wikipedia.org/wiki/Italic-type.

[10] Instructions to authors, Emerg Infect Dis. On wwwnc. Cdc.goc/eid/scientific-nomenclature.

[11] G. Chen, X. Zhang, X. Zeng. Survey of 13 cases of melioidosis in southern China. Chin J Zoonoses. 11 (199552-53). (in Chinese with abstract in English)

[12] A. Whitmore, C.S. Krishnaswami. An account of the discovery of a hitherto undescribed infectious disease among the population of Rangoon. Indian Med Gazette. 47(1912): 262-267

[13] E. Yabuuchi; Y. Kosako, H. Oyaizu, I. Yano, H. Hotta, Y. Hashimoto, T. Ezaki, M. Arakawa. Proposal of Burkholderia gen. nov. and transfer of seven species of the genus Pseudomonas homology group II to the new genus, with the type species Burkholderia cepacia (Palleroni and Holmes 1981) comb. nov.". Microbiol Immunol. 36 (1992): 1251-75.

[14] M. McCormick. Rats, Communications, and Plague: Toward an Ecological History. J Interdiscip History. 34 (2003): 1-25.

[15] J. Borges, F.T. Tegtmeier, N.T. Padron, M. C. Mueller, E. M. Lang, G. B. Stark. Chorioallantoic membrane angiogenesis model for tissue engineering: a new twist on a classic model. Tissue Eng. 9(2003):441-50.

[16] Information for Authors, Research Online, Microbiology Society. http://www.microbiologyresearch.org/authors/information-for-authors. 